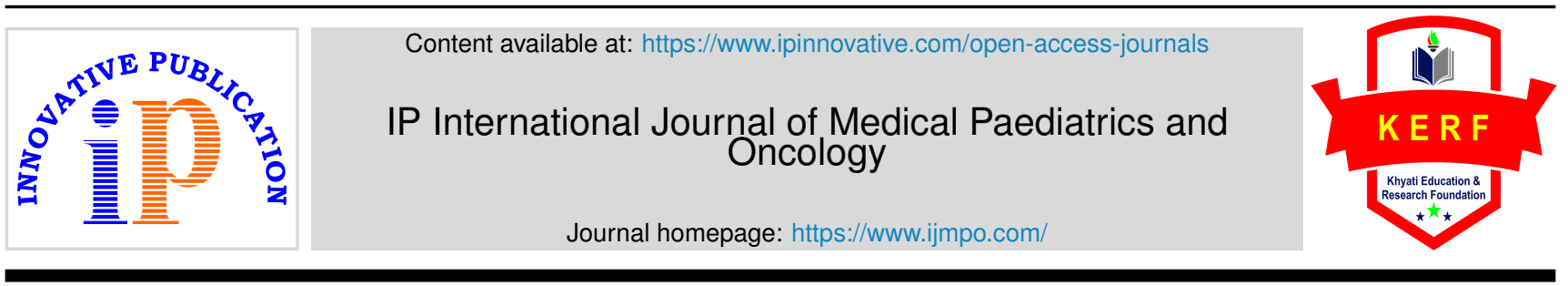

Original Research Article

\title{
Enteric fever among febrile children aged 1 to 18 years: Prevalence, diagnostic performance of the Typhoid dot IgM and antibiotic multi-drug resistance
}

\author{
Ramesh Dasari ${ }^{1}$, K Swapna Reddy ${ }^{1, *}$, Sivani Saraswathi Kuruvada ${ }^{1}$, \\ Alekhya Nimmagadda ${ }^{1}$, Tahera Tabussum ${ }^{1}$, S Srikrishna ${ }^{1}$ \\ ${ }^{1}$ Dept. of Paediatrics, Apollo Institute of Medical Sciences and Research, Jubilee Hills, Hyderabad, Telangana, India
}

\section{A R T I C L E I N F O}

\section{Article history:}

Received 02-02-2021

Accepted 18-02-2021

Available online 25-03-2021

\section{Keywords:}

Salmonella Typhi

Enteric Fever

Widal Test

Typhidot

\begin{abstract}
A B S T R A C T
Introduction: Typhoid is a bacterial infection that can lead to a high fever, diarrhoea, and vomiting. It can be fatal. It is caused by the bacteria Salmonella typhi. Globally, around 21.5 million people a year contract typhoid. If typhoid is caught early, it can be successfully treated with antibiotics; if it is not treated, typhoid can be fatal.

Materials and Methods: This is a retrospective and observational study conducted in Department of Pediatrics from the 1 January 2016 to 30 December 2019. Epidemiological data, clinical features, blood culture, Typhidot IgM test and other laboratory parameters from all the patients were collected and it was analyzed by using the Statistical package for social sciences (SPSS) version 21 Fisher's exact test.

Results:During the study period total 290 febrile patients with symptoms suggestive of Enteric fever were included. Incidence of enteric fever among these 290 patients was $32.41 \%(n=94)$, of which laboratory confirmed culture positive incidence was $17.09 \%(\mathrm{n}=55)$ and probable cases of enteric fever based on positive serodiagnosis where culture showed no growth was $17.09 \%(n=39)$. Out of 290 patients, 170 $(58.62 \%)$ were male and rest $120(41.37 \%)$ were female. The serological positivity by Widal test in this study was $32.06 \%$ (n=93/290). Serodiagnosis by Typhidot IgM was $27.24 \%(n=79 / 290)$.

Conclusions: High rate of MDR S. Typhi is alarming and suggests need for better living standards, health education, correct diagnosis and proper treatment as control strategies.

(C) This is an open access article distributed under the terms of the Creative Commons Attribution License (https://creativecommons.org/licenses/by/4.0/) which permits unrestricted use, distribution, and reproduction in any medium, provided the original author and source are credited.
\end{abstract}

\section{Introduction}

Typhoid fever is endemic disease and is a major health problem in developing countries and its diagnosis on clinical ground is difficult. Children are disproportionately affected by typhoid fever, with peak incidence long known to occur in individuals aged 5 to $<15$ years of age. ${ }^{1}$ It is a systemic infection caused by Salmonella typhi, usually through ingestion of contaminated food or water (faecal-oral route). A similar but often less severe disease, paratyphoid fever, is caused by Salmonella paratyphi A, B or C. ${ }^{2}$ The often-nonspecific presentation of typhoid fever makes clinical diagnosis difficult as it may be confused with a wide

\footnotetext{
* Corresponding author.

E-mail address: sapymbbs@gmail.com (K. S. Reddy).
}

range of other common febrile illnesses in regions where typhoid fever is endemic. ${ }^{3}$

There are no $100 \%$ sensitive or ideal tests available for enteric fever. The sensitivity of a single blood culture is approximately $60 \%$ and is affected by the volume of blood obtained for culture. ${ }^{4}$ Sensitivity of blood culture is further reduced by the common practice of starting treatment with antibiotics prior to confirmation of the diagnosis. Blood culture is not always available and, when it is, it takes at least 2 to 3 days. ${ }^{5}$ As a result, diagnosis may be delayed or overlooked and patients without typhoid fever may receive unnecessary and inappropriate antimicrobial treatment. For this reason, typhoid rapid antibody tests can facilitate diagnosis and disease management. ${ }^{6}$ 
Typhidot IgM is widely used serological test. In latest, a Cochrane database systematic review study has been published in May 2017. It has conducted a meta-analysis of 37 studies with total 5080 participants (mostly from Asia) with the aim to assess the diagnostic accuracy of commercially available rapid diagnostic tests (RDTs) and prototypes for detecting Salmonella typhi or paratyphi. A infection in symptomatic persons living in endemic areas. It concluded that these tests had moderate diagnostic accuracy. Across all forms of the Typhidot test, the average sensitivity was $84 \%$ (95\% CI $73 \%$ to $91 \%$ ) and specificity was $79 \%$ (95\% CI $70 \%$ to $87 \%)^{7}$

\section{Aim and Objectives}

The objectives of this study were to study the prevalence of enteric fever in children admitted to pediatric ward with acute febrile illness aged 2 to 15 years and compare the sensitivity, specificity, positive predictive value (PPV) and negative predictive value (NPV) of Typhidot IgM compared to Bactec blood culture which is the gold standard for diagnosis of enteric fever and to analyze the effect of antibiotic taken prior to testing, on sensitivity and specificity of Typhidot IgM and Bactec blood culture and also study to the emergence of antibiotic multidrug resistance.

\section{Materials and Methods}

This is a retrospective and observational study conducted in Department of Pediatrics from the 1 January 2016 to 30 December 2019.

\subsection{Inclusion criteria}

The children aged 1 year to 18 years with clinically compatible enteric fever (The acute illness with highgrade fever $(95 \%)$, coated tongue $(76 \%)$, anorexia $(70 \%)$, vomiting (39\%), hepatomegaly (37\%), diarrhea (36\%), toxicity $(29 \%)$, abdominal pain $(21 \%)$ with blood leukocyte counts are frequently low in relation to the fever and toxicity) with either Typhidot IgM or blood culture positive for Salmonella species were sampled and analyzed.

\subsection{Exclusion criteria}

Children with alternative proven diagnosis or incomplete details were excluded.

Epidemiological data, clinical features, blood culture, Typhidot IgM test and other laboratory parameters from all the patients were collected and it was analyzed by using the

\subsection{Statistical analysis}

Statistical package for social sciences (SPSS) version 21 Fisher's exact test. P-value of less than 0.05 was considered as statistically significant. The study was conducted after getting ethical approval from the Apollo Institute of Medical
Sciences and Research, Jubilee Hills, Hyderabad.

\section{Results}

During the study period total 290 febrile patients with symptoms suggestive of Enteric fever were included. Incidence of enteric fever among these 290 patients was $32.41 \% \quad(n=94)$, of which laboratory confirmed culture positive incidence was $17.09 \%(n=55)$ and probable cases of enteric fever based on positive serodiagnosis where culture showed no growth was $17.09 \%(n=39)$.

In Table 1, out of 290 patients, $170(58.62 \%)$ were male and rest $120(41.37 \%)$ were female.

In Table 2, age of the participants ranged from 2 years to 15 years and $60 \%$ study population were less than 29 years old $(n=162 / 290)$ with mean age of 8.51 years. The results of enteric fever cases and its association with sociodemographic factors were shown in Table 2.

\subsection{Widal tube agglutination test}

The serological positivity by Widal test in this study was $32.06 \%(n=93 / 290)$. Whereas blood culture showed only $55(17.09 \%)$ febrile patients as culture confirmed cases, with one Widal false negative test result (Table 3). The sensitivity, specificity, positive predictive value (PPV) and negative predictive value (NPV) of Widal test were $95.75 \%$, $76.27 \%, 49 \%$ and $97.02 \%$ respectively as compared with the gold standard test of blood culture (Table 4).

\subsection{Typhidot test}

Serodiagnosis by Typhidot IgM was 27.24\% (n=79/290) (table-3). The sensitivity, specificity, PPV and NPV for Typhidot IgM test were $100 \%, 88.88 \%, 70.24 \%$ and $100 \%$ respectively (Table 4 ).

In Table 6 , the patterns of susceptibility for S. typhi and S. paratyphi were comparable and the overall rate of antibiotic resistance to three or more agents was $52.1 \%$.

\section{Discussion}

Typhoid is a bacterial infection that can lead to a high fever, diarrhoea, and vomiting. It can be fatal. It is caused by the bacteria Salmonella typhi. The infection is often passed on through contaminated food and drinking water, and it is more prevalent in places where handwashing is less frequent. ${ }^{8}$ Globally, around 21.5 million people a year contract typhoid. If typhoid is caught early, it can be successfully treated with antibiotics; if it is not treated, typhoid can be fatal. ${ }^{9}$

The current study identified higher seropositivity $(32.41 \%)$ while lower prevalence from blood culture $(17.09 \%)$. The varied positivity rate among serodiagnosis and blood culture may be because of difference in time of sample collection, false positivity in Widal test and 
Table 1: Distribution of gender of Suspected Febrile Patients (n-290) and in Laboratory Diagnosed Enteric Fever Cases (n=94) and their Association

\begin{tabular}{lcccc}
\hline Gender & $\begin{array}{c}\text { Total Numbers Tested } \\
(\mathbf{n = 2 9 0})\end{array}$ & $\begin{array}{c}\text { Enteric Fever Positive } \\
(\mathbf{n = 9 4 )}\end{array}$ & \% Prevalence & p - value \\
Male & 170 & 54 & 31.7 & $\mathbf{0 . 0 9 3 2}$ \\
Female & 120 & 40 & 33.3 & \\
\hline
\end{tabular}

Table 2: Distribution of age of Suspected Febrile Patients (n-290) and in Laboratory Diagnosed Enteric Fever Cases (n=94) and their Association

\begin{tabular}{lcccc}
\hline Age & $\begin{array}{c}\text { Total Numbers } \\
\text { Tested }(\mathbf{n = 2 9 0})\end{array}$ & $\begin{array}{c}\text { Enteric Fever Positive } \\
(\mathbf{n = 9 4 )}\end{array}$ & \% Prevalence & p - value \\
$\mathbf{2 - 5}$ yrs. & 79 & 31 & 39.2 & 19.2 \\
6-9 yrs. & 83 & 16 & 26.8 & $\mathbf{0 . 0 0 3 2}$ \\
$\mathbf{1 0 - 1 2}$ yrs. & 67 & 18 & 47.5 & \\
$\mathbf{1 3 - 1 5}$ yrs. & 61 & 29 & \\
\hline
\end{tabular}

Table 3: Comparison of Incidence of Enteric Fever by Laboratory Diagnosis by Blood Culture, Widal Test, and Typhidot Test

\begin{tabular}{llll}
\hline $\begin{array}{l}\text { Laboratory Investigations } \\
(\mathbf{n = 2 9 0 )}\end{array}$ & Organisms Identified & No. of Positive Results & \% Incidence \\
Blood culture & S. Typhi & 49 & 16.89 \\
Widal tube agglutination & S. Paratyphi A & 6 & 2.06 \\
test & S. Typhi & $95 \mathrm{TO}(\geq 1: 80)+\mathrm{TH}(\geq 1: 160)$ & 32.75 \\
& & $=93$ TO $(\geq 1: 80)=6$ & 4.1 \\
Typhidot IgM test & S. Paratyphi A & $12 \mathrm{TO}(\geq 1: 80)+\mathrm{AH}(\geq 1: 160)$ & 27.24 \\
\hline
\end{tabular}

Table 4: Comparative evaluation of widal test and typhidot test as compared with blood culture

\begin{tabular}{lcc}
\hline Parameters & Widal Test & Typhidot \\
Sensitivity & $95.75 \%$ & $100 \%$ \\
Specificity & $76.27 \%$ & $88.88 \%$ \\
Positive Predictive Value & $49 \%$ & $70.24 \%$ \\
Negeitive Predictive Value & $97.2 \%$ & $100 \%$
\end{tabular}

Table 5: Clinical features of patients evaluated for typhoid fever

Parameter
Fever
Headache
Anorexia
Diarrhea
Discomfort /pain
Tachycardia
Vomiting
Constipation Abdominal
Hepatomegaly
Pallor
Splenomegaly
Relative bradycardia
Jaundice

Typhoid suspects $\mathbf{N}=\mathbf{2 9 0} \mathbf{n}(\boldsymbol{\%})$
$290(100.0)$
$229(79.1)$
$198(68.3)$
$168(58.0)$
$155(53.3)$
$145(49.9)$
$137(47.4)$
$46(16.0)$
$45(15.4)$
$33(11.5)$
$16(5.6)$
$9(2.8)$
$7(2.3)$

Table 6: Patterns of antibiotic multi-drug resistance (MDR) in isolates from febrile patients with typhoid fever, as confirmed by culture

\begin{tabular}{lccc} 
MDR pattern & S. Typhi $(\mathbf{N}=\mathbf{3 1}) \mathbf{n}(\boldsymbol{\%})$ & S. Paratyphi $(\mathbf{N}=\mathbf{6 3}) \mathbf{n}(\boldsymbol{\%})$ & Total $(\mathbf{N}=\mathbf{9 4}) \mathbf{n}(\boldsymbol{\%})$ \\
R3 & $6(19.0)$ & $16(25.0)$ & $22(23.4)$ \\
R4 & $4(12.9)$ & $5(7.9)$ & $9(9.5)$ \\
R5 & $3(9.5)$ & $9(14.2)$ & $12(12.7)$ \\
R6 & $1(3.2)$ & $3(4.7)$ & $4(4.2)$ \\
R7 & $1(3.2)$ & $1(1.5)$ & $2(2.1)$ \\
Total & $15(48.3)$ & $34(53.9)$ & $49(52.1)$ \\
\hline
\end{tabular}


local endemicity of enteric fever with patients having raised antibody titre from old diseases. Blood tests when gathered past 7-10 days history of fever, had lesser opportunity to give blood culture positive report, while Widal seropositivity expanded. Likewise, Widal false negative report with blood culture and Typhidot IgM test positive report was a because of fever was 5-7 days.

Widal false positive is additionally normal and could exist due to infection with other Enterobacteriaceae with cross responding immune response or Malaria. In addition, in our study, Widal test however commonly utilized as normal screening test, it had low specificity (76.27\%) and PPV (49\%) in our investigation showing high false positive revealing. Comparable examinations by Felgner $\mathbf{J}$ et al. likewise indicated lower affectability, explicitness, PPV, NPV of Widal tests. ${ }^{10}$ Therefore, rapid tests with better sensitivity and specificity are required for the correct determination of enteric fever.

The Typhidot IgM test is a rapid dot-enzyme immune assay that gets positive in enteric fever as early as in the first week of fever. It had high sensitivity $(100 \%)$ and sensitivity $(88.88 \%)$ with preferred execution over Widal test in our investigation. Comparative investigaion by Dittrich $\mathrm{S}$ et al. likewise demonstrated that Typhidot-M was better than Widal test in their analytic affectability and particularity. ${ }^{11}$

More febrile patients were under 6-9 years age group; however, the burden of enteric fever was most extreme under 2-5 years age group and it is the solitary significant risk factor related with prevalence of enteric fever (by Chi-square test, $\mathrm{p}=0.0032,95 \%$ CI-Confidence Interval) (Table 2). Superior expectations for everyday comforts with great water source, improved disinfection and better cleanliness gauges alongside right determination and legitimate treatment may add to control endemicity of enteric fever by conceivably disposing of fecal transporters and shedders from the populace. Additionally, expanding pace of MDR S. Typhi is disturbing and proposes need for wellbeing schooling and successful control methodologies.

The most common clinical features in our patients with positive typhoid cultures were fever, abdominal pain/ discomfort, anorexia, headache, diarrhoea, vomiting and tachycardia. However, only anorexia, constipation, abdominal pain/discomfort, splenomegaly and relative bradycardia showed statistically significant differences when compared with the clinical features of febrile patients with negative typhoid cultures. In accordance with our observations, fever (90-100\%), headache $(50-98 \%)$ and anorexia $(65-90 \%)$ were the most consistent findings among typhoid fever patients in other settings. ${ }^{12-15}$ Although diarrhoea (19-56\%), abdominal pain (11-48\%) and vomiting (20-46\%) were commonly reported buy other studies, the proportions of individuals with these symptoms were lower than that seen in our current study. ${ }^{16-19}$

\section{Conclusion}

The high rate of MDR S. Typhi isolation recommends the requirement for proper AST reporting and safely utilization of antibiotics agents for treating enteric fever. These measures are fundamental to prevent rise and spread of XDR S. Typhi, again changing enteric fever into a lethal and untreatable condition. Despite the fact that blood culture is the highest quality level test for its conclusion, the quantity of culture affirmed enteric fever cases is low. Along these lines, we regularly rely upon serodiagnosis. For right determination of enteric fever, quick serological tests with better sensitivity and specificity are required. Likewise, better expectations for everyday comforts, wellbeing schooling, and right treatment are needed to control endemicity of enteric fever.

\section{Conflicts of Interest}

All contributing authors declare no conflicts of interest.

\section{Source of Funding}

None.

\section{References}

1. MacFadden DR, Bogoch II, Andrews JR. Advances in diagnosis, treatment, and prevention of invasive Salmonella infections. Curr Opin Infect Dis. 2016;29(5):453-8. do1:10.1097/qco.000000000000302

2. Parry CM, Wijedoru L, Arjyal A, Baker S. The utility of diagnostic tests for enteric fever in endemic locations. Expert Rev Anti Infect Ther . 2011;9(6):711-25. 101:10.1586/eri. 147

3. Andrews JR, Ryan ET. Diagnostics for invasive Salmonella infections: Current challenges and future directions. Vaccine. 2015;33:C8-C15. do1:10.1016/].vaccine.2015.02.030.

4. Klemm EJ, Shakoor S, Page AJ. Emergence of an extensively drug-resistant Salmonella enterica serovar typhi clone harboring a promiscuous plasmid encoding resistance to fluoroquinolones and third-generation cephalosporins. Lancet Glob Health. 2016;9:516-7.

5. Schroeder LF, Elbireer A, Jackson JB, Amukele TK. Laboratory Diagnostics Market in East Africa: A Survey of Test Types, Test Availability, and Test Prices in Kampala, Uganda. Plos One. 2015;10(7):e0134578. do1:10.1371/journal.pone.0134578.

6. Khanam F, Sheikh A, Sayeed MA. Evaluation of a Typhoid/ Paratyphoid diagnostic assay (TPTest) detecting anti-Salmonella IgA in secretions of peripheral blood lymphocytes in patients in Dhaka. PLoS Negl Trop Dis. 2013;7:2316-2316.

7. Kalckreuth VV, Konings F, Aaby P. The typhoid fever surveillance in Africa program (TSAP): clinical, diagnostic, and epidemiological methodologies. Clin Infect Dis. 2016;62:9-16.

8. Das S, Rajendran K, Dutta P, Saha TK, Dutta S. Validation of a new serology-based dipstick test for rapid diagnosis of typhoid fever. Diagn Microbiol Infect Dis . 2013;76(1):5-9. do1:10.1016/].diagmicrobio.2013.01.012

9. Islam K, Sayeed MA, Hossen E. Comparison of the performance of the TPTest, Tubex, Typhidot and widal immunodiagnostic assays and blood cultures in detecting patients with typhoid fever in Bangladesh, including using a Bayesian latent class modeling approach. PLoS Negl Trop Dis. 2016;10:4558.

10. Felgner J, Jain A, Nakajima R, Liang L, Jasinskas A, Gotuzzo E, et al. Development of ELISAs for diagnosis of acute typhoid fever in Nigerian children. PLoS Negl Trop Dis . 2017;11(6):e0005679. doi:10.1371/journal.pntd.0005679 
11. Bruxvoort KJ, Leurent B, Chandler CIR. The impact of introducing malaria rapid diagnostic tests on fever case management: a synthesis of ten studies from the act Consortium. Lancet Global Health. 2017;97:310-23.

12. Dittrich S, Tadesse BT, Moussy F. Target product profile for diagnostic assay to differentiate between bacterial and non-bacterial infections and reduce antimicrobial overuse in resource-limited settings: an expert consensus. PLoS One. 2016;11:161721-33.

13. Denkinger CM, Dolinger D, Schito M, Wells W, Cobelens F, Pai M, et al. Target Product Profile of a Molecular Drug-Susceptibility Test for Use in Microscopy Centers. J Infect Dis . 2015;211(suppl_2):S39_ S49. doi:10.1093/infdis/jiu682.

14. Das JK, Hasan R, Zafar A. Trends, associations, and antimicrobial resistance of Salmonella typhi and paratyphi in Pakistan. Am J Trop Med Hyg. 2018;99:48-54.

15. Ghani AC, Burgess DH, Reynolds A, Rousseau C. Expanding the role of diagnostic and prognostic tools for infectious diseases in resourcepoor settings. Nat. 2015;528(7580):S50-2. 10i:10.1038/nature 6038

16. Mogasale V, Ramani E, Mogasale VV, Park JY. What proportion of Salmonella Typhi cases are detected by blood culture? A systematic literature review. Ann Clin Microbiol Antimicrob . 2016;15(1):32.

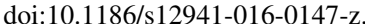

17. Storey HL, Huang Y, Crudder C, Golden A, Santos T, Hawkins K, et al. A Meta-Analysis of Typhoid Diagnostic Accuracy Studies: A Recommendation to Adopt a Standardized Composite Reference. PLoS One. 2015;10(11):e0142364. doi:10.1371/1ournal.pone.0142364

18. Obaro SK, Tam PYI, Mintz ED. The unrecognized burden of typhoid fever. Expert Rev Vaccines. 2017;16(3):249-60. d01:10.1080/14760584.201/. 252553
19. Thriemer K, Ley B, Menten J, Jacobs J, Ende J. A systematic review and meta-analysis of the performance of two point of care typhoid fever tests, Tubex TF and Typhidot, in endemic countries. PLoS One. 2013;8(12)::e81263. do1:10.1371/journal.pone.0081263

\section{Author biography}

Ramesh Dasari, Associate Professor

K Swapna Reddy, Assistant Professor

Sivani Saraswathi Kuruvada, Intern

Alekhya Nimmagadda, Intern

Tahera Tabussum, Post Graduate

S Srikrishna, Professor and HOD

Cite this article: Dasari R, Reddy KS, Kuruvada SS, Nimmagadda A, Tabussum T, Srikrishna S. Enteric fever among febrile children aged 1 to 18 years: Prevalence, diagnostic performance of the Typhoid dot IgM and antibiotic multi-drug resistance. IP Int J Med Paediatr Oncol 2021;7(1):41-45. 